\title{
ON THE BOUND FOR A PAIR OF CONSECUTIVE QUARTIC RESIDUES OF A PRIME
}

\author{
R. G. BIERSTEDT AND W. H. MILLS
}

It is easy to show that every prime $p$ greater than 5 has a pair $n, n+1$ of positive consecutive quadratic residues not exceeding 10 . Furthermore, any prime $p$, such as 43 , for which $2,3,5,7$ are all quadratic nonresidues has 9,10 as the smallest such pair. M. Dunton [1] has shown that every prime $p$, except 2,7 , and 13 , has a pair $n, n+1$ of positive consecutive cubic residues not exceeding 78 , and that there exist an infinite number of primes for which 77,78 is the smallest such pair.

In this paper we prove the analogous result for quartic residues. ${ }^{1}$

THEOREM. Every prime p, except 2, 3, 5, 13, 17, 41, has a pair $n, n+1$ of positive consecutive quartic residues not exceeding 1224, 1225. Furthermore, there exist an infinite number of primes $p$ for which 1224, 1225 is the smallest such pair.

Proof. If $p \equiv 3(\bmod 4)$, then all the quadratic residues of $p$ are quartic residues, and the result follows from the known result for quadratic residues. Hence we may suppose $p \equiv 1(\bmod 4)$. Let $g$ be a primitive root modulo $p$. Let $\chi$ be the quartic character modulo $p$ defined by $\chi(n)=i^{b}$ if $n \equiv g^{b}(\bmod p)$. Then $n$ is a quartic residue of $p$ if and only if $\chi(n)=1$.

Suppose $p$ has no pair of positive consecutive quartic residues less than 1226. Then for every integer $N, 1 \leqq N \leqq 1224$, we have either $\chi(N) \neq 1$ or $\chi(N+1) \neq 1$. Setting $N=1$ we obtain $\chi(2) \neq 1$, and setting $N=80$ we obtain $\chi(5)=\chi(80) \neq 1$. Thus $\chi(2)=-1$, $i$, or $-i$; and $\chi(5)=-1, i$, or $-i$. Without loss of generality we suppose $\chi(2)=-1$ or $i$. Furthermore, if $\chi(2)=-1$, we may suppose that $\chi(5)=-1$ or $i$. This leads to five cases:

Case I. $\chi(2)=\chi(5)=-1$. Putting $N=9$ we obtain $\chi(3) \neq 1,-1$. Without loss of generality we suppose $\chi(3)=i$. The argument indicated by Table I now eliminates this case.

Case II. $\chi(2)=-1, \chi(5)=i$. Setting $N=3$ we obtain $\chi(3) \neq 1$, and setting $N=15$ we obtain $\chi(3) \neq-i$. Therefore $\chi(3)=-1$ or $i$. Thus

Presented to the Society, January 9,1961, under the title On the existence of a bound for a pair of consecutive quartic residues modulo a prime; received by the editors May 1, 1962.

1 The results for fifth and sixth powers have been obtained by electronic computing machines [4]. 
we have two subcases which are eliminated by Table II.

\section{TABLE I}

The case $\chi(2)=\chi(5)=-1, \chi(3)=i$

\begin{aligned} \hline \hline$N &$ Conclusion \\ \hline 288 & $\chi(17) \neq 1,-1 \\ 255 & \chi(17) \neq i \\ & \chi(17)=-i \\ 51 & \chi(13) \neq 1 \\ 25 & \chi(13) \neq-1 \\ 39 & \chi(13) \neq-i \\ & \chi(13) \neq i \\ 195 & \chi(7) \neq 1,-1 \\ & \chi(49)=-1 \\ 40 & \chi(41) \neq 1 \\ 81 & \chi(41) \neq-1 \\ 245 & \chi(41) \neq i \\ & \chi(41)=-i \\ 287 & \chi(7) \neq i \\ & \chi(7)=-i \\ 10 & \chi(11) \neq 1 \\ 21 & \chi(11) \neq-1 \\ 77 & \chi(11) \neq i \\ 594 & \chi(11) \neq-i \\ & \end{aligned}$

TABLE II

The case $\chi(2)=-1, \chi(5)=i$

\begin{tabular}{cc|cc}
\hline \multicolumn{2}{c|}{ Subcase A: $\chi(3)=-1$} & \multicolumn{2}{|c}{ Subcase B: $\chi(3)=i$} \\
\hline$N$ & Conclusion & $N$ & Conclusion \\
\hline 49 & $\chi(7) \neq 1,-1$ & 288 & $\chi(17) \neq 1,-1$ \\
35 & $\chi(7) \neq-i$ & 50 & $\chi(17) \neq-i$ \\
& $\chi(7)=i$ & & $\chi(17)=i$ \\
675 & $\chi(13) \neq 1,-1$ & 49 & $\chi(7) \neq 1,-1$ \\
728 & $\chi(13) \neq i$ & 119 & $\chi(7) \neq-i$ \\
64 & $\chi(13) \neq-i$ & 168 & $\chi(7)=i$ \\
& & 441 & $\chi(13) \neq 1,-1$ \\
& & 64 & $\chi(13) \neq i$ \\
& & &
\end{tabular}

Case III. $\chi(2)=i, \chi(5)=-1$. Putting $N=15$ we get $\chi(3) \neq-1$, and putting $N=24$ we get $\chi(3) \neq i$. Therefore $\chi(3)=1$ or $-i$. These sub- 
cases are eliminated by Table III.

TABLE III

The case $\chi(2)=i, \chi(5)=-1$

\begin{tabular}{cc|cc}
\hline \multicolumn{2}{c|}{ Subcase A: $\chi(3)=1$} & \multicolumn{2}{|c}{ Subcase B: $\chi(3)=-i$} \\
\hline$N$ & Conclusion & $N$ & \multicolumn{1}{c}{ Conclusion } \\
\hline 48 & $\chi(7) \neq 1,-1$ & 6 & $\chi(7) \neq 1$ \\
224 & $\chi(7) \neq-i$ & 35 & $\chi(7) \neq-1$ \\
& $\chi(7)=i$ & 20 & $\chi(7) \neq i$ \\
168 & $\chi(13) \neq 1,-1$ & & $\chi(7)=-i$ \\
675 & $\chi(13) \neq i,-i$ & 16 & $\chi(17) \neq 1$ \\
& & 84 & $\chi(17) \neq-1$ \\
& & 119 & $\chi(17) \neq i$ \\
& & 255 & $\chi(17) \neq-i$ \\
\hline
\end{tabular}

Case IV. $\chi(2)=\chi(5)=i$. Putting $N=15$ we have $\chi(3) \neq-i$. Thus we have three subcases here-these are eliminated by Table IV.

TABLE IV

The case $\chi(2)=\chi(5)=i$

\begin{tabular}{|c|c|c|c|c|c|}
\hline \multicolumn{2}{|c|}{ Subcase $A: \chi(3)=1$} & \multicolumn{2}{|c|}{ Subcase B: $\chi(3)=-1$} & \multicolumn{2}{|c|}{ Subcase C: $\chi(3)=i$} \\
\hline$N$ & Conclusion & $N$ & Conclusion & $N$ & Conclusion \\
\hline 48 & $\begin{aligned} \chi(7) & \neq 1,-1 \\
\chi(49) & =-1\end{aligned}$ & $\begin{array}{r}12 \\
624\end{array}$ & $\begin{array}{l}x(13) \neq 1 \\
x(13) \neq-1\end{array}$ & $\begin{array}{r}16 \\
255\end{array}$ & $\begin{array}{l}\chi(17) \neq 1 \\
\chi(17) \neq-1\end{array}$ \\
\hline 16 & $x(17) \neq 1$ & 675 & $x(13) \neq i,-i$ & 135 & $x(17) \neq i$ \\
\hline 1224 & $x(17) \neq i$ & & & & $x(17)=-i$ \\
\hline 255 & $x(17) \neq-i$ & & & $\begin{array}{r}374 \\
99\end{array}$ & $\begin{array}{l}\chi(11) \neq 1 \\
\chi(11) \neq-1\end{array}$ \\
\hline 169 & $\chi(13) \neq 1,-1$ & & & 54 & $\chi(11) \neq-i$ \\
\hline 26 & $\begin{array}{l}\chi(13) \neq-i \\
\chi(13)=i\end{array}$ & & & 384 & $\begin{aligned} \chi(11) & =i \\
\chi(7) & \neq-1\end{aligned}$ \\
\hline 120 & $\chi(11) \neq 1,-1$ & & & 84 & $\chi(7) \neq i$ \\
\hline 935 & $\chi(11) \neq i$ & & & 35 & $x(7) \neq-i$ \\
\hline \multirow[t]{4}{*}{143} & $x(11) \neq-i$ & & & & $x(7)=1$ \\
\hline & & & & 168 & $x(13) \neq 1,-1$ \\
\hline & & & & 220 & $\chi(13) \neq i$ \\
\hline & & & & 39 & $\chi(13) \neq-i$ \\
\hline
\end{tabular}

Case V. $\chi(2)=i, \chi(5)=-i$. This last case is eliminated by Table V. We have now shown that every prime $p$, except $2,3,5,13,17,41$, 
has a pair of consecutive positive quartic residues not exceeding 1224 , 1225.

TABLE V

The case $\chi(2)=i, \chi(5)=-i$

\begin{aligned} \hline \hline$N &$ Conclusion \\ \hline 9 & $\chi(3) \neq 1,-1 \\ 15 & \chi(3) \neq i \\ & \chi(3)=-i \\ 6 & \chi(7) \neq 1 \\ 35 & \chi(7) \neq i \\ 224 & \chi(7) \neq-i \\ & \chi(7)=-1 \\ 168 & \chi(13) \neq 1,-1 \\ 624 & \chi(13) \neq i \\ & \chi(13)=-i \\ 16 & \chi(17) \neq 1 \\ 255 & \chi(17) \neq-1 \\ 135 & \chi(17) \neq i \\ & \chi(17) \neq-i \\ 10 & \chi(11) \neq 1 \\ 99 & \chi(11) \neq-1 \\ 33 & \chi(11) \neq i \\ 351 & \chi(11) \neq-i \\ & \end{aligned}$

It follows from Theorem 3 of [2] that there exist an infinite number of primes $p$ such that, with appropriate choice of primitive root $g$, $\chi(3)=1$ and $\chi(q)=i$ for all other primes $q$ less than 1226 . Let $p$ be such a prime. Then $\chi(n)$ is determined for all $n$ such that $1 \leqq n \leqq 1225$. The only odd values of $n$ in this range for which $\chi(n)=1$ are

$$
n=1,3,9,27,81,243,625,729,875,1225 .
$$

On the other hand $\chi(n) \neq 1$ for

$$
n=2,4,8,10,26,28,80,82,242,244,624,626,728,730,874,876 \text {, }
$$

and $\chi(1224)=1$. Hence 1224,1225 is the smallest pair of positive consecutive quartic residues of $p$. Thus there are an infinity of primes $p$ for which 1224,1225 is the smallest pair of consecutive quartic residues. This completes the proof of the theorem.

The primes $5,13,17$, and 41 occurred in the factorizations of the numbers used in the proof. Hence no conclusion can be drawn from this proof concerning them. However a brief calculation shows that these primes do not have pairs of consecutive quartic residues. 
It is known from a theorem of A. Brauer [3] that every sufficiently large prime $p$ has a pair of consecutive quartic residues. Our result shows that this is true for all primes $p$ greater than 41 . Brauer's proof does not establish the existence of an upper bound for the least pair of consecutive quartic residues of $p$.

D. H. and Emma Lehmer [5] have shown that there is no bound for three consecutive positive quadratic residues. In other words there exist primes for which the smallest triplet $n, n+1, n+2$ of consecutive positive quadratic residues is arbitrarily large. The same result is therefore true for three consecutive quartic residues.

\section{REFERENCES}

1. M. Dunton, $A$ bound for consecutive pairs of cubic residues (to appear).

2. W. H. Mills, Characters with preassigned values, Canad. J. Math. 15 (1963), 169-171.

3. A. Brauer, Über Sequenzen von Potenzresten, S.-B. Preuss Akad. Wiss. Phys.Math. K1. (1928), 9-16.

4. D. H. Lehmer, Emma Lehmer and W. H. Mills, Pairs of consecutive power residues, Canad. J. Math. 15 (1963), 172-177.

5. D. H. Lehmer and Emma Lehmer, On runs of residues, Proc. Amer. Math. Soc. 13 (1962), 102-106.

Colorado College and YALE UNIVERSITY 